\title{
Las consecuencias de la violencia filio-parental reflejadas en una historia de vida
}

\author{
The consequences of child-parent violence reflected in a life story \\ José Jesús TRUJILlo VARGAS \\ Corporación Universitaria del Caribe, Colombia \\ trujilo215@hotmail.com \\ Marta SAHAGÚN NAVARRO \\ Corporación Universitaria del Caribe, Colombia \\ martasn2000@hotmail.com \\ Rocío CÁRDENAS RODRÍGUEZ \\ Universidad Pablo de Olavide, Sevilla, España \\ mrcarrod@upo.es \\ Andrés Fernando RAMíREZ GIRALDO \\ Corporación Universitaria del Caribe, Colombia \\ andres.ramirez@cecar.edu.co
}

Recibido: $12 / 11 / 2014$

Revisado: $19 / 12 / 2014$

Aceptado: 25/11/2015

Disponible on line: 20/01/2016

\section{Resumen}

Los cambios existentes en la familia, unidos a un proceso de transformación social, han posibilitado que exista un fenómeno relativamente poco conocido como es el de la violencia ascendente, que se erige como una de las modalidades de violencia que más se vienen dando en dicho ámbito. En base al estudio de este fenómeno, en esta investigación hemos utilizado la técnica cualitativa de la historia de vida, apoyándonos en un diario de campo donde hemos tomado notas sobre nuestro quehacer diario a nivel terapéutico, en aras de mitigar los efectos de dicho proceso. En esta investigación se establecieron los siguientes objetivos: establecer la conexión que existe entre estilo educativo familiar y el uso de la violencia por parte del menor y evaluar en qué medida la terapia familiar mitiga el uso de la violencia por parte del menor. El modelo educativo familiar, junto a otras dimensiones ${ }^{1}$, determina que las situaciones de violencia se den de manera reiterada entre padres e hijos, existiendo refuerzos negativos continuos de ambas partes para mantener un ciclo recurrente de conductas, del que difícilmente se puede «salir» si no es a través de un proceso de terapia psicológica continuado.

Palabras clave: menor, padres, violencia, modelo educativo, terapia familiar.

\begin{abstract}
A process of social transformation allied with ongoing changes to the family has made possible the existence of a relatively little-known phenomenon: that of child-parent violence, which is raised as one of the most commonly experienced forms of violence in the family environment. Based on the study of this phenomenon, in our research we have used the qualitative technique of a life story, making use of a field diary in which we have taken notes on our daily work in the therapeutic context, for the purposes of mitigating the effects of such a process. The following research objectives were set: establishing the connection existing between family education style and the use of violence by the minor; and evaluating the extent to which family therapy mitigates the use of violence by the minor. The family education model, together with other dimensions, results in situations of child-parent violence occurring repeatedly, with continuing negative reinforcement from both parties in order to maintain a recurrent cycle of conduct, from which it is difficult to «escape» other than through a process of ongoing psychological therapy.
\end{abstract}

Keywords: minor, parents, violence, education model, family therapy.

Referencia normalizada: Trujillo Vargas, J. J., Sahagún Navarro, M., Cárdenas Rodríguez, R. y Ramírez Giraldo, A. F. (2016): «Las consecuencias de la violencia filio-parental reflejadas en una historia de vida». Cuadernos de Trabajo Social, 29(1): $119-128$.

Sumario: Introducción. 1. Metodología. 2. Resultados. 3. Conclusiones. 4. Referencias bibliográficas.

${ }^{1}$ Como pudieran ser la hiperreactivad vs infrarreactividad (Santiago, 2008) de los padres en la relación con sus hijos. 


\section{Introducción}

En la crisis económica actual, la institución familiar se ha visto profundamente afectada. Hay varios factores que influirán de forma decisiva en el grado de vulnerabilidad de las familias, incluyendo el tipo de empleo o el desempleo de los padres, los problemas de salud, el conflicto generacional e intergeneracional, el abandono escolar, etc. (Segado, Osca y López, 2013, p. 254).

En los últimos años, se han intensificado los esfuerzos por comprender los factores generales relacionados con la violencia juvenil. Guarda estrecha relación con la evolución que ha experimentado la opinión pública en las últimas décadas, con respecto a este fenómeno, en la que se puede percibir una preocupación creciente por los signos de violencia en jóvenes y adolescentes, en etapas cada vez más tempranas (Agustina y Romero, 2013). Este fenómeno no es un proceso que pueda considerarse extraño, ya que lo mismo ocurrió con los demás tipos de violencia intrafamiliar. Tanto el maltrato infantil como el conyugal son situaciones ancladas, desde hace muchos años, en el seno de la familia. Ha sido a raíz de su definición como inadecuados y dañinos, unido al esfuerzo de sacar los casos a la luz, lo que ha modificado la visión fragmentada que se tenía sobre ellos, favoreciendo la emergencia social de un problema oculto. De igual forma, la violencia filio-parental permanecía encubierta, como uno más de los conflictos que presentaba una familia con otras disfuncionalidades (Pereira y Bertino, 2009).

Tradicionalmente, este tipo de violencia ha sido encubierta por las víctimas y desmentida por los agresores (Aroca, 2010), lo que dificulta determinar la prevalencia de la misma. Entre 2000 y 2004, se aprecia un aumento de las denuncias de los adultos que han padecido dicha violencia, como queda recogido en los distintos informes de la Fiscalía General (Pereira y Bertino, 2009). Sin embargo, la Memoria de la Fiscalía del Estado (2010), anuncia un descenso de estas denuncias.

Las investigaciones realizadas sobre este fenómeno reflejan que las edades en que los hijos agreden a sus progenitores oscilan entre $\operatorname{los} 4 \mathrm{y}$ los 24 años, conformando los 11 años la edad media de inicio. Todos los estudios destacan la adolescencia como período crítico para la manifestación de tales comportamientos (Pérez y Pereira, 2006).

De manera específica, en este estudio nos hemos centrado en algunos de los factores que determinan el uso de la violencia como manera de convivencia. Muy especialmente nos interesa el ámbito familiar como punto de partida para explicar este tipo de conductas, ya que la disfuncionalidad en este ámbito es una de las causas principales para que, posteriormente, los menores desarrollen conductas violentas en otras relaciones fuera de la familia. Analizamos la violencia ascendente, a través de las propuestas teóricas sobre la terapia en violencia filio-parental llevada a cabo por autores como Pereira (2006), Santiago (2008) y Pereira y Bertino (2009, 2010), partiendo del estudio de un caso con el que se intervino terapéuticamente, a fin de dar a conocer las causas y consecuencias que existen en el uso de la violencia familiar (desde dentro), concretamente en el de la violencia filio-paren$\mathrm{tal}^{2}$, que se define como el conjunto de conductas reiteradas de agresiones físicas: golpes, empujones, arrojar objetos; verbales: insultos repetidos, amenazas; o no verbales:-gestos amenazadores, ruptura de objetos apreciados; dirigidas a los padres o adultos que ocupan su lugar (Pereira, 2006). El aspecto unánime de este tipo de violencia, en los autores consultados, es que se trata de una violencia física, verbal y emocional por parte del hijo hacia sus padres (Pérez y Pereira, 2006; Santiago, 2008; Aroca, 2010).

Existen autores que han diferenciado la violencia intrafamiliar ascendente como un fenómeno específico y con entidad propia dentro del campo que nos ocupa (Harbin y Madden, 1979; Sears, Maccoby y Levin, 1957, citados por Walsh y Krienert, 2007; Paterson, Luntz y Perlesz, 2002), haciendo referencia a aquellos actos de violencia y agresión que los menores llevan a cabo sobre sus familiares ascendentes y, en especial, contra sus progenitores.

En el estudio terapéutico de este fenómeno, Henry, Harbin y Madden (1979, citados por Pereira y Bertino, 2009) advirtieron la disfunciona-

2 Este estudio se centra específicamente en la influencia de los modelos familiares en el uso de la violencia y en las dimensiones que hacen que esos modelos sean más o menos extremos. Dimensiones descritas por Santiago (2008). 
lidad que presentan las familias, inmersas en el proceso de violencia filio-parental, en tres de las principales áreas de la dinámica familiar: a) organización jerárquica y de señalamiento de normas; b) protección de la imagen familiar; y c) separación y fusión. En estrecha relación con estas áreas disfuncionales, y coincidiendo con Santiago (2008), se puede decir que en el trabajo cotidiano que se ha venido llevando a cabo con los padres de los adolescentes conflictivos, se han observado dos dimensiones que tienen una poderosa influencia para entender el conflicto que se genera entre padres e hijos adolescentes. La primera dimensión se refiere a la existencia de la dicotomía exigencia versus permisividad, en la relación entre padres e hijos; mientras la otra dimensión se refiere al apego versus desapego que los progenitores muestran en la relación cotidiana con sus descendientes. Combinando ambas dimensiones en un eje de coordenadas, saldrían cuatro tipos diferentes de padres en su relación con los hijos, que quedan representados en la Figura $1^{3}$.

Estas dimensiones, unidas al modelo educativo que posea cada familia ${ }^{4}$, van a determinar, cómo se gestionan los conflictos en cada contexto familiar y si, a partir de esta gestión, surgen procesos violentos o no. Para evaluar el alcance de estos conflictos y si los mismos terminan en un proceso de violencia entre padres e hijos, conviene establecer otra diferenciación tipológica, en base también a otras cuatro dimensiones: hiperreactividad (entendida como aquella reacción enérgica ante la situación convivencial) e infrarreactividad (entendida como aquella ausencia de reacción) por parte de los padres, y por otro lado, descontrol e inhibición, ante las respuestas de los padres por parte de los hijos. Como queda recogido en la Figura $2^{5}$.

Autores como Peek y Fisher (1985), en su estudio sobre violencia familiar y estilos educativos, correlacionaron la violencia filio-parental con cuatro estilos educativos: a) estilo punitivo y estricto; b) estilo estricto pero no punitivo; c) estilo no punitivo ni estricto; y d) estilo violento, mostrando una clara relación entre los estilos: violento (aquel generalizado en la familia, ejercido de manera habitual) y punitivo, pero no estricto (es decir, más arbitrario y sin normas claras), con la violencia filio-parental.

Para Musitu y García (2004, citados en Estévez, Jiménez y Musitu, 2007), los estilos educa-

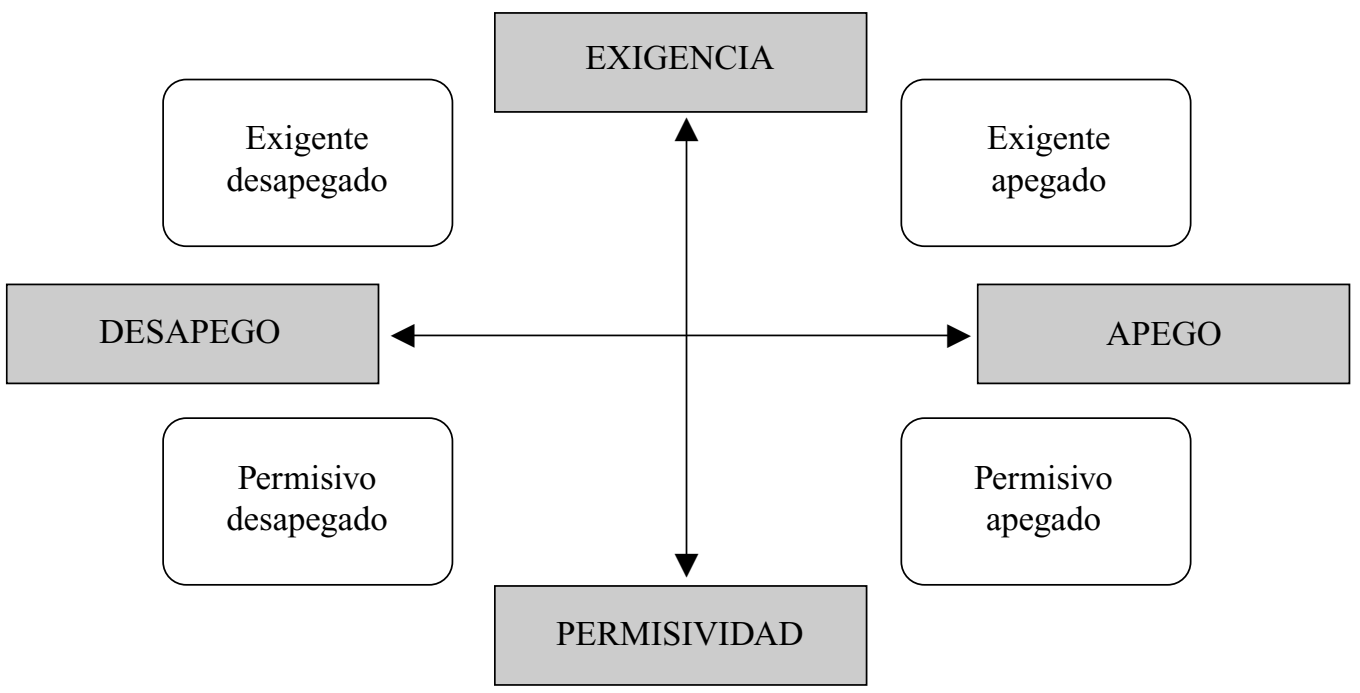

Figura 1. Exigencias versus permisividad, apego vs desapego.

Fuente: Adaptado de Santiago (2008).

${ }^{3}$ Atendiendo a estas dimensiones, podemos encontrar cuatro perfiles de padres, que se recogen en el trabajo de Santiago (2008).

${ }^{4}$ Siguiendo la tipología descrita por Nardone (2003).

5 Dichos parámetros van a desencadenar distintas respuestas de interacción entre padres e hijos como queda descrito en el trabajo de Santiago (2008). 
HIPERREACTIVIDAD

(progenitor)
4. Complementariedad rígida

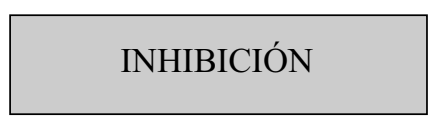

(hijo)

3. Vacío relacional

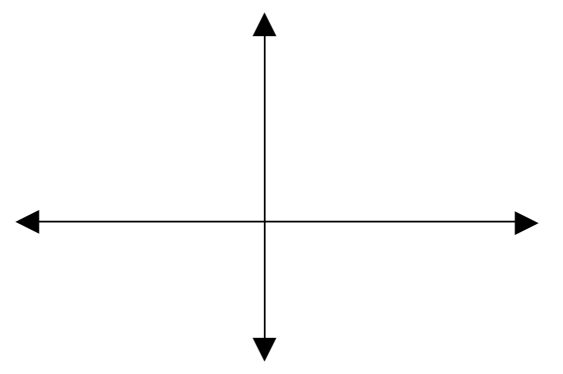

INFRARREACTIVIDAD
1. Escalada simética

(hijo)

2. Posicionamiento ambivalente

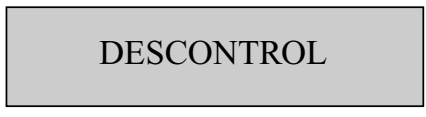

Figura 2. Dimensiones de patrón relacional paterno-filial. Fuente: Adaptado de Santiago (2008).

tivos orientados hacia el afecto y la implicación de los padres (estilos permisivo e indulgente) son más eficaces que los estilos orientados hacia la coerción (estilos autoritario y negligente) que pretenden que los hijos desarrollen competencias psicológicas y sociales adecuadas, y por otro lado, la utilización y el ejercicio del razonamiento conduce a una mayor comprensión e interiorización de las normas sociales que la mera imposición o la permisividad total.

\section{Metodología}

La metodología utilizada para esta investigación es cualitativa, se ha usado la técnica historia de vida, que se ha ido elaborando mediante la recogida de datos de un total de cuarenta y cinco entrevistas psicoterapéuticas, que se han realizado durante cuatro años y medio. En los primeros seis meses de intervención la periodicidad de las entrevistas era semanal, posteriormente mensual y en la última etapa anual, a través de sesiones telefónicas (a fin de evaluar si existían situaciones de recaída). Aunque en la primera etapa, el menor acudió a terapia (durante dos sesiones), el grueso de las mismas se ha llevado a cabo con la madre, a través de una serie de entrevistas semiestructuradas ad hoc, en las que la información se recogía durante el propio proceso de terapia (in situ) a través de un diario de campo, en el que se iba anotando, pormenorizadamente, cada uno de los aspectos reseñables de la situación de violencia filio-parental existente.
A medida que se iba construyendo la historia de vida con esta persona, que era revisada continuamente tanto por los terapeutas que intervenían en el caso (2), como por sus protagonistas. De igual modo, esta persona dio a conocer la misma a varias personas de su entorno, antes de validarla definitivamente. Una demostración más de que si no es a través del lenguaje de los propios protagonistas, y en su marco de referencia, no se podría llegar a conocer lo que sienten, piensan y padecen estas personas (Trujillo, 2013a).

Aunque se trata de un caso, es representativo, teniendo en cuenta la casuística de este fenómeno. Existiendo una serie de características de la personalidad del menor, en este caso, que aparecen con mayor frecuencia en los agresores: baja autoestima, egocentrismo, impulsividad y ausencia o disminución de la capacidad empática y consumo de tóxicos (Pereira y Bertino, 2009). A lo que se une una disparidad de criterios por parte de los padres a la hora de interponer consecuencias a las conductas de su hijo y el desapego por parte de uno de los progenitores, como ocurre en muchos de los casos de violencia filioparental (Pereira 2006; Pereira y Bertino, 2009; 2010).

En este caso la madre mantenía una relación fusional con sus dos hijos, aunque se hacía mucho más patente con el hijo menor, lo que se retroalimentaba por el hecho de que su marido, en muchas ocasiones, se mostraba frío, distante o ausente (Pereira y Bertino, 2009). Esto se agudi- 


\begin{tabular}{|c|c|c|c|}
\hline Objetivos & Dimensiones & Subdimensiones & Variables \\
\hline $\begin{array}{l}\text { 1. Establecer la } \\
\text { conexión que } \\
\text { existe entre estilo } \\
\text { educativo familiar } \\
\text { y uso de la violen- } \\
\text { cia por parte del } \\
\text { menor. }\end{array}$ & $\begin{array}{l}\text { Modelo educativo } \\
\text { familiar y rela- } \\
\text { ción con violencia } \\
\text { ejercida por parte } \\
\text { del menor. }\end{array}$ & $\begin{array}{l}\text { Modelo educativo } \\
\text { familiar en el que } \\
\text { se está desenvol- } \\
\text { viendo el menor y } \\
\text { cómo afecta en } \\
\text { las conductas del } \\
\text { mismo. }\end{array}$ & $\begin{array}{l}\text { - Qué modelo impera (Nardone, } \\
\text { 2003; Santiago, 2008): hiperprotector, } \\
\text { permisivo, autoritario, sacrificante, de- } \\
\text { legante o intermitente. } \\
\text { - Tipología parental (exigencia vs } \\
\text { permisividad, apego vs desapego). } \\
\text { - Tipología parento- filial (Santiago, } \\
\text { 2008): hiperreactividad vs infrarreacti- } \\
\text { vidad, inhibición vs descontrol. } \\
\text { - Qué fomenta la semilla patológica } \\
\text { en la relación familiar. } \\
\text { - Establecimiento de límites y nor- } \\
\text { mas en las relaciones familiares. }\end{array}$ \\
\hline $\begin{array}{l}\text { 2. Evaluar en qué } \\
\text { medida la terapia } \\
\text { familiar mitiga el } \\
\text { uso de la violen- } \\
\text { cia por parte del } \\
\text { menor. }\end{array}$ & $\begin{array}{l}\text { Terapia familiar } \\
\text { como posibilidad } \\
\text { de filtraje del pro- } \\
\text { ceso violento. }\end{array}$ & $\begin{array}{l}\text { Valoración por } \\
\text { parte de los dife- } \\
\text { rentes miembros } \\
\text { de la familia so- } \\
\text { bre la utilidad de } \\
\text { la terapia familiar. }\end{array}$ & $\begin{array}{l}\text { - Qué manifiestan los diferentes } \\
\text { miembros de la familia sobre la terapia } \\
\text { familiar llevada a cabo. } \\
\text { - En qué medida las personas impli- } \\
\text { cadas gestionan el conflicto de manera } \\
\text { diferente, a raíz de la terapia. } \\
\text { - Descenso o no de la violencia filio- } \\
\text { parental. }\end{array}$ \\
\hline
\end{tabular}

Cuadro 1. Objetivos, dimensiones y variables del estudio.

Fuente: Elaboración propia.

zó tras la separación conyugal. Este tipo de relación fusional, de uno de los progenitores, suele ocurrir en muchas ocasiones en este tipo de casos (Pereira y Bertino, 2009).

Los objetivos concretados en dimensiones, subdimensiones y variables de esta historia vida quedan reflejados en el Cuadro 1. A tenor de los mismos, se analizan y detallan los resultados del estudio en el siguiente apartado.

\section{Resultados}

\subsection{Antecedentes del caso}

Esta historia de vida trata de una madre que, durante años, ha soportado humillaciones, vejaciones y maltrato de todo tipo por parte de su hijo, desde que éste tenía 14 años hasta poco antes de llegar a la adultez, aunque el relato comienza cuando el hijo problemático tenía 15 años. Por ello acudió a Servicios Sociales, y de ahí fue derivado a un servicio de intervención en violencia familiar, en el que se intervino con ella durante dos años de manera intensiva y, posteriormente, durante otros dos años y medio se realizó un seguimiento telefónico (para evaluar posibles recaídas). En la actualidad, el hijo es mayor de edad y vive en la misma ciudad que su madre, pero de forma independiente, manteniendo una relación cordial con la misma.

En los inicios de la terapia, la protagonista de la historia de vida narraba su situación de la siguiente forma:

Antes de la separación, había mal ambiente en casa, existían muchas discusiones entre mi entonces marido y yo, así que tras varios años decidimos separarnos. Mi ex-marido siempre ha tenido una línea de actuación con mi hijo y yo otra totalmente distinta, con lo cual nuestro hijo trataba de aprovecharse de la situación. Él conseguía todo lo que quería de su padre y éste le hacía multitud de regalos, sin embargo yo pretendía que me respetará porque soy su madre y que valorara que las cosas materiales tenía que ganárselas con una conducta positiva. Un buen día, el padre dejó de hacerle regalos y fue cuando comenzaron los conflictos entre ellos.

Como la situación de violencia verbal (amenazas, chantajes, insultos, etc., y contra el mobiliario de casa) era insostenible y no podía controlar la situación, denunció a su hijo por maltrato y el menor fue internado en un centro de reforma, durante dos períodos distintos. 
La relación entre la protagonista de la historia de vida y su hija mayor era bastante positiva, sin embargo, ésta apenas tenía relación con su padre y en tan sólo una ocasión le había visitado, después de la separación conyugal. Con su hermano mantenía frecuentes disputas, aunque la lejanía geográfica hacía que no coincidieran en muchas ocasiones en el mismo espacio.

\subsection{Resultados a tenor de los objetivos}

Teniendo en cuenta los objetivos del Cuadro 1, podemos extraer los siguientes resultados:

\section{a) Objetivo número 1}

Cabe destacar que en contextos donde el menor no podía controlar ni manipular las interacciones con otros adultos de referencia se comportaba de una forma más adaptada y apropiada (como ocurrió en el centro de reforma en el que estuvo internado, a raíz de la denuncia interpuesta por su madre), controlando en mayor medida la frustración proveniente del no conseguir lo que quería en cada momento (como así ocurría en el ámbito familiar). Los padres solían permitir y tolerar unos niveles altos de violencia por parte de los hijos antes de tomar medidas (Pérez y Pereira, 2006), lo que no ocurre, por ejemplo, en los centros de reforma, donde las consecuencias de las conductas violentas suelen ser inmediatas.

Se observó que el padre llevaba a cabo un modelo educativo intermitente extremo (en ocasiones se mostraba muy permisivo y en otras ocasiones exigente) con su hijo, en donde no existían unas peticiones coherentes hacia él (en muchas ocasiones contradictorias). Por otro lado, el padre arrastraba la frustración por no haber superado la separación de su ex-mujer, a la par de que poseía una adicción severa al alcohol (que no estaba siendo tratada por ningún profesional), lo que repercutía sobre su manera de comportarse y sobre la manera de tratar a su hijo, quien manipulaba la situación frecuentemente, consiguiendo todo aquello que se proponía.

El padre intentaba conseguir el beneplácito de sus hijos con regalos, porque nunca había sido capaz de crear un vínculo afectivo realmente fuerte con ninguno de ellos, y durante el tiempo que vivieron juntos entorpecía constantemente la labor de la madre, que era bastante más coherente (como así lo atestiguaba el propio menor). La madre lo relataba de la siguiente forma:
Mi hijo cogió la costumbre de llamar a su padre para conseguir lo que quería cuando yo no accedía a sus pretensiones, ya fueran unos pantalones, una camisa, unos zapatos deportivos... Intenté comunicarme con el padre para que no le comprara cosas y decirle que de esta manera fomentaba un carácter negativo en el niño y perjudicial para él, pero la comunicación fue imposible en todo momento. Él pensaba que de esta manera se lo ganaba más.

Se trata de un padre permisivo, desapegado, por lo tanto estaba «desenganchado emocionalmente» de su hijo (el menor comenta que sólo va a casa del padre por el beneficio material que le reporta, cuando se siente culpable) y la madre se empeñaba en compensar esto, con un intento de exigencia y de apoyo emocional a su hijo; por lo tanto se muestra como apegada, permisiva (a pesar de que sus demandas no las llevaba a cabo su hijo, al final cedía a sus chantajes) e hiperprotectora (ya que en última instancia, siempre perdonaba las acciones violentas del menor). Este tipo de padres declaran que quieren a sus hijos por encima de todo, renunciado a la conducta coercitiva (Pereira y Bertino, 2009).

Por otra parte, el modelo intermitente del padre y la infrarreactividad ante las situaciones conflictivas con el hijo, posibilitaban que el menor ostentara un mayor poder en el hogar paterno, exigiendo continuamente que el padre satisficiera todos sus deseos materiales. Y cuando el padre no aguantaba más la situación, la infrarreactividad se transformaba en hiperreactividad para tratar de que el menor corrigiera su conducta. Lo que posibilitaba un mayor descontrol en el hijo, que se traducía en frecuentes escaladas simétricas entre ambos, que en muchas situaciones acaban en violencia del menor contra el padre. En estas situaciones, la hostilidad parental genera hostilidad filial (Omer, 2004). El progenitor intenta controlar, exigir, modificar o inhibir la conducta-problema de su hijo. Éste reacciona enfrentándose, rebelándose o haciendo caso omiso. Esto provoca que el progenitor lo siga intentando, a través del convencimiento de que debe seguir con su conducta controladora (más de lo mismo), pues, de no ser así, el hijo cada vez estaría peor. Este ciclo disfuncional interactivo lleva a dramáticas situaciones de crisis, que pueden desembocar en la violencia física (Santiago, 2008), como ya se había dado en ocasiones. 
Existiendo un posicionamiento ambivalente con la madre, contra quien arremetía el hijo arremetía cuando no conseguía lo que quería, y un vacío relacional con el padre, que se caracterizaba porque el padre no sabía comunicarle al hijo su interés por sus inquietudes, expectativas, intereses, etc., y éste expresaba poco sobre los mismos, dándose un distanciamiento emocional entre ambos.

La semilla patológica en esta relación a tres bandas residía en que, cuando el menor presionaba para conseguir lo que quería, no era correspondido por su madre; sin embargo el padre sí que accedía a todas sus pretensiones, con lo cual el menor cada vez tenía más poder tanto en el hogar de la madre como en el del padre (al que finalmente dejó de acudir de forma periódica). Todo ello con la pretensión del menor de aumentar el poder y la dominación dentro del núcleo familiar, o sea, con la obtención de beneficios secundarios (Pereira y Bertino, 2009).

Los límites en la convivencia entre hijo y padre no se encontraban definidos y, con el tiempo, se había extendido generalizado a la convivencia con la madre. Por lo que se puede considerar como un padre excesivamente permisivo, ya que estaba convencido de la importancia de tener una relación simétrica con su hijo desde muy temprana edad (Cyrulnik, 2005; Pereira y Bertino, 2009).

En algunas ocasiones, la madre, aunque establecía límites en la relación con su hijo, también accedía a sus pretensiones, por el miedo que sentía al haber vivido situaciones violentas con anterioridad. Como señalan Cotrell y Monk (2004), algunos progenitores son reticentes a comunicar su situación porque tienen miedo a que ello produzca futuros y aún más graves incidentes de violencia en el hogar. En algunos casos, la preocupación se basaba en las amenazas de sus hijos o en la experiencia vivida de incremento de su violencia, tras habérselo comunicado a los profesionales o allegados.

Durante su estancia en el centro, se mostró totalmente afable y comenzó a ganar privilegios dentro del mismo al poco tiempo (según su madre). Esta aparente estabilidad conductual sólo se veía alterada con las llamadas telefónicas a la madre, donde continuaba exigiéndole que retirara la denuncia, y otros privilegios materiales. Igualmente, cuando ésta lo visitaba al centro existían situaciones de chantajes continuos.
Según el relato de la madre:

Un día, tuvimos una discusión en donde él me seguía exigiendo cosas materiales para que se las llevase al centro. Después de ese capítulo hubo un altercado en donde él se mordió, según me decía para ponerse en mi lugar y porque me había causado daño. A medida que se iban agotando los tres meses iba teniendo más incertidumbre, porque en realidad no sabía lo que iba a pasar, si iba a volver a casa o si iba a seguir en el centro tres meses más. Tenía una sensación rara, porque por un lado quería que volviera pero por otro, y por lo hablado con él, me daba cuenta de que mi hijo había progresado poco.

Lo que da a entender que el menor era consciente de su actitud, pero quería seguir obteniendo beneficios materiales, aunque para ello tuviera que utilizar la violencia.

\section{b) Objetivo número 2}

En reiteradas ocasiones, la madre nos ha comunicado que sin la terapia no hubiera podido solventar a nivel emocional este proceso, en donde han existido tantos altibajos ni hubiera podido presentar la denuncia por el maltrato del que estaba siendo víctima por parte de su hijo. Lo que choca frontalmente con la consideración que poseía de la terapia familiar al inicio de la intervención (había tenido algunas experiencias negativas a nivel terapéutico).

Por su parte, el menor (actualmente mayor de edad), siempre ha sido reacio a las intervenciones terapéuticas, ya que suponían la asunción de su responsabilidad en todo este proceso de violencia ascendente (habiendo pasado por varios procesos terapéuticos, con distintos psicólogos). La hermana no consideraba que con terapia su hermano pudiera tener grandes cambios en su manera de actuar (como así dio a conocer en una sesión que acompañó a su madre). Ella no había estado tan influenciada por los altibajos emocionales de su padre, ya que la relación era casi inexistente y desde hacía tiempo vivía fuera del ámbito familiar. Con su madre sí existía un apego importante y en ocasiones compartían tiempo de ocio juntas, aún a pesar de vivir en diferentes ciudades.

El padre no participó en un proceso terapéutico continuado, porque no se encontraba en condiciones de salud propicias para una intervención cognitivo-conductual, aunque en varias 
ocasiones fue contactado por teléfono, considerando que era importante y beneficioso que su hijo pudiera acudir a terapia.

Aunque el menor, tras su paso por el centro de reforma, había continuado con actitudes de chantaje, amenazas y manipulación, su madre se mostraba menos permisiva ante las mismas, a raíz del proceso terapéutico, y comenzó a ser consciente de que su hiperprotección reforzaba la conducta de su hijo. Lo que se hizo patente, al igual que en el caso presentado por Pereira y Bertino (2010), cuando la madre relató que la terapia le había ayudado a ser consciente de muchas actitudes erróneas que ella tenía, como por ejemplo estar pendiente en exceso de sus hijos.

A pesar de todos los acontecimientos comentados en torno al uso de la violencia por parte del menor contra su madre, en la actualidad ese pasado no está condicionando, de manera determinante, al presente, ya que ambos tienen una relación normalizada, promovida por el hecho de que tienen vidas independientes. La madre vive con su pareja, mientras el menor ha encontrado trabajo (tras el intento fallido de estudiar una carrera) y vive solo en un piso de alquiler.

\section{Conclusiones}

La violencia puede constituir un estilo relacional aprendido que parte de una reconstrucción individual inadecuada de procesos interpersonales donde se dan episodios de agresividad. Ello requiere que dicha agresividad instintiva haya propiciado efectos deseables para el sujeto que la ejerce, aprendiendo que mediante su uso puede volver a conseguirlos (Estalayo, 2011, p. 388).

Como se ha podido comprobar en esta historia de vida y en otras investigaciones (Santiago, 2008; Trujillo, 2013b), el no reconocimiento de las emociones en su vida personal, es lo que posibilita que los menores vivan en un ciclo recurrente permanente de frustraciones de las que no encuentran una salida, más allá de los actos agresivos y por lo que se encuentran constantemente a la defensiva cuando los adultos les piden responsabilidades. Dicho reconocimiento lo interpretan como una situación de fragilidad ante sus iguales y ante los mayores.

En referencia a los modelos educativos, significar que Nardone (2003) y Santiago (2008), consideran que no existe un estilo educativo que favorezca la violencia, sino que este fenómeno dependerá de las dimensiones anteriormente expuestas (hiperreactividad versus infrarreactividad; inhibición versus descontrol). A actitudes más extremas por parte de los padres, a la hora de disipar las conductas negativas de sus hijos, mayor probabilidad de que estos extremen las mismas, dándose de esta forma situaciones de violencia filio-parental. Esto se hace patente tanto en este caso como en otros estudios relacionados (Santiago, 2008; Trujillo, 2012; Trujillo, 2013a; Trujillo, 2013b).

Micucci (1995) y Omer (2004) consideran que, una vez que inicia el ciclo de la violencia, los hijos se orientan cada vez más hacia el poder, mientras los padres lo hacen hacia la indefensión. Esto hace que los padres se centren en los aspectos negativos de la relación con sus hijos, disminuyendo la atención y el reconocimiento de las cualidades positivas que sus hijos evidencian en la interacción con ellos, lo que también queda demostrado en esta investigación.

Teniendo en cuenta todo lo descrito en esta historia de vida, y siguiendo a Gallagher (2004a; 2004b), en este tipo de intervenciones terapéuticas se ha de intentar reducir y redistribuir la culpabilidad que sienten los padres y que aprendan a clarificar las normas a través del establecimiento de consecuencias a las conductas violentas de sus hijos. Respecto al menor, se debe pretender que no tenga conductas violentas, dándole mayor importancia a la responsabilidad de los menores que a su protección, puesto que si se le da más importancia a la protección, la responsabilidad que se le exige disminuye, favoreciendo la aparición de conductas de abuso.

Una de las limitaciones más importantes que presenta este estudio es el hecho de que, al no poderse realizar intervención terapéutica, de manera regular, con el menor, con la hermana y con el padre, la historia de vida no se ha podido complementar con las aportaciones, vivencias e interpretaciones de los otros miembros familiares, lo que no quiere decir, por otra parte, que esté exento de rigurosidad. Se trata de un estudio pormenorizado desde el interior de un caso en donde la violencia filio-parental presentaba patrones relacionales, a nivel familiar, de difícil reconducción.

Esta historia de vida se presenta como una invitación para seguir investigando y comprobando las distintas teorías que explican este fenómeno, 
en aras de redefinir aquellos factores que intervienen en dicho proceso, a medida que el ámbito familiar y social va cambiando. E igualmente se presenta como un estudio a tener en cuenta para que tanto padres e hijos, como los distintos agentes sociales, reflexionen y analicen las diferentes situaciones, relacionadas con la violencia familiar, que encuentren en su quehacer cotidiano.

\section{Referencias bibliográficas}

Agustina, J.R. y Romero, F. (2013). Análisis criminológico de la violencia filio-parental. Revista de Derecho Penal y Criminología, 9, 225-266.

Aroca, C. (2010). La violencia filio-parental: una aproximación a sus claves. Valencia: Universidad de Valencia.

Cyrulnik, B. (2005). El amor que nos cura. Barcelona. Gedisa.

Cottrell, B. y Monk, P. (2004). Adolescent-to-parent abuse. A qualitative overview of common themes. Journal of family Issues, 25(8), 1072-1095.

Estalayo, A. (2011). Violencia adolescente y circularidad: De la teoría a la práctica. En R. Pereira (Coord.), Adolescentes en el siglo XXI. Entre impotencia, resiliencia y poder (pp.385-402). Madrid: Morata.

Estévez, E., Jiménez, T. y Musitu, G. (2007). Relaciones entre padres e hijos adolescentes. Valencia: Nau Llibres.

Fiscalía General del Estado. (2010). Memoria elevada al gobierno de S. M. Recuperado de https://www.fiscal.es/fiscal/PA_WebApp_SGNTJ_NFIS/descarga/MEMFIS11.PDF?idFile=b2eb 5133-c29e-4930-a783-41e4b15fc70f

Gallagher, E. (2004a). Parents Victimised by their Children. Australian and New Zeland Journal of Family Therapy, 25, 1-12.

Gallagher, E. (2004b). Youth who victimised their parents. Australian and New Zeland Journal of Family Therapy, 25, 94-105.

Micucci, J. (1995). Adolescents who assault their parents: A family systems approach to treatment. Psychotherapy, 32(1), 154-161.

Nardone, G. (2003). Modelos de familia: conocer y resolver los problemas entre padres e hijos. Barcelona: Herder.

Omer, H. (2004). Non violent resistance: a new approach to violent and self-destructive children. Cambridge: Cambridge University Press.

Paterson, R., Luntz, H., Perlesz, A. y Cotton, S. (2002). Adolescent violence towards parents: Maintaining family connections when the going gets tough. Australian and New Zealand Journal of Family Therapy, 23, 90-100.

Peek, W.; Fisher, J. y Kidwell, J. (1985). Teenage violence toward parents: A neglected dimension of family violence. Journal of marriage and the family, 47, 1051-1058.

Pereira, R. (2006). Violencia filio-parental, un fenómeno emergente. Revista Mosaico, 36, 7-8.

Pereira, R. y Bertino, L. (2009). Una comprensión ecológica de la violencia filio-parental. Redes, 21, 69-90.

Pereira, R. y Bertino, L. (2010). Cuando los adolescentes toman el poder. Un caso de violencia filioparental. Sistemas Familiares, 1, 93-115.

Pérez, T. y Pereira, R. (2006). Violencia filio-parental: revisión de la bibliografía. Revista Mosaico, $36,10-17$.

Santiago, L. (2008). Intervención con familias de adolescentes problemáticos. Barcelona: Centro Kine.

Segado, S., Osca, A., y López, A. (2013). Trabajo Social con familias y conflicto familia-trabajo. Cuadernos de Trabajo Social, 26(2), 253-264.

Trujillo, J. J. (2012). El culpable no era yo. RES, 15, 1-10.

Trujillo, J. J. (2013a). Menores en riesgo: la violencia de los menores en diferentes contextos socioeducativos. Sevilla: Universidad Pablo de Olavide.

Trujillo, J. J. (2013b). Menor imputado, menor selectivamente perdonado. En M.C. Pérez y M.M. Molero (coords.), Variables Psicológicas y Educativas para la intervención en el ámbito escolar (pp. 501-506). Almería: Asociación Universidad de Educación y Psicología. 
Walsh, J. y Krienert, J. (2007). Child-parent violence: An empirical analysis of offender, victim, and event characteristics in a national sample of reported incidents. Journal of Family Violence, 22, 563-574. 